\title{
Continuous Fascia Iliaca Compartment Block for Post Operative Analgesia in Fracture Femur
}

Bigen man Shakya, ${ }^{1}$ Ninadini Shrestha ${ }^{1}$

${ }^{1}$ Department of Anaesthesiology, Tribhuvan university Teaching hospital, Maharajgunj, Kathmandu, Nepal.

\section{ABSTRACT}

The postoperative pain management in the elderly is challenging due to comorbidities and change in physiology due to age itself. This limit the use of medication which include pain medication also. The fascia iliaca compartment block has been described in the literature for fracture of femur. It has even been safely used by non anaesthesiologist also. To our knowledge, we did not find any case report of continuous fascia iliaca compartment block published in Nepal. This is our first experience of successful continous fasicia iliaca compartment block in case of 89 year old lady with multiple co morbidities in whom traditional pain medication might be difficult to use. We encourage to practice this block which is both safe and easy to perform with good results.

Keywords: Continous fascia iliaca compartment block.

\section{INTRODUCTION}

The single shot fascia iliaca block is used for analgesia in fracture femur. With the development of catheter, continous fascia iliaca block has been used in literature. Despite proven efficacy, it is never tried in our institute. We like to share a first case in our institute and hope to continue this .

\section{CASE DETAILS}

A 87 year old lady with diagnosis of left Intertrochanteric fracture of hip was posted for elective surgery, fixation with dynamic hip screw. On pre-anaesthetic evaluation, she had history of coronary artery disease with hypertension controlled with medication. Her medication were tab aspirin $50 \mathrm{mg} \mathrm{OD}$, tab atorvastatin $10 \mathrm{mg} \mathrm{OD}$, tab losartan $25 \mathrm{mg} \mathrm{OD}$, tab monotrate 10 $\mathrm{mg}$ bd, tab diltiazeam 30mg, tab lasix 20mg. Her recent ECG and echocardiography showed no major abnormality. On the second day of admission during routine blood investigation the serum potassium was found to be $7.0 \mathrm{mmol} / \mathrm{L}$ without any ECG changes. The serum creatinine was $2.08 \mathrm{mg} / \mathrm{dl}$. Serum sodium was $131 \mathrm{mmol} / \mathrm{L}$. The repeat serum potassium was 6.8 $\mathrm{mmol} / \mathrm{L}$. The 24 hour urine output was $1000-1500 \mathrm{ml}$. The physician made the diagnosis of drug induced hyperkalemia and tab Lasix was hold and they started treatment for hyperkalemia. Inspite of repeated medical therapy, the potassium level remained high. So the nephrologist decided to do hemo dialysis (Heparin free) for refractory hyperkalemia. The post dialysis showed serum creatinine of $1.62 \mathrm{mg} / \mathrm{dl}$ and serum sodium 128 $\mathrm{mmol} / \mathrm{L}$ and serum potassium $5.3 \mathrm{mmol} / \mathrm{L}$.

The surgery was performed under spinal anaesthesia. The duration of surgery was one hour and there was no adverse introperative event. We decided to perform continous infusion in fascia iliac compartmrnt with $0.25 \%$ ropivacine for postoperative pain management. We inserted epidural catheter in fascia iliaca compartment under ultrasound guidance. Under aseptic precaution the transducer of USG was placed in upper region of thigh transversely below inguinal ligament. After visualization of femoral vessels and nerve the transducer was moved laterally. The fascia lata, fascia iliaca and iliacus muscle were visualized. The touhy needle was inserted by in plane technique. After the fascia iliaca has been pierced, $30 \mathrm{ml}$ of $0.25 \%$ ropivacine was pushed. With this volume the space between the fascia lata and iliacus muscle was demarcated and epidural catheter was inserted under USG visualization. The catheter was fixed at $6 \mathrm{~cm}$ and sutured with skin to secure. $40 \mathrm{ml}$ of 0.25 $\%$ Ropivacine was given and $5 \mathrm{ml} / \mathrm{hr}$ of $0.25 \%$ ropivacine was infused via syringe pump. The NRS(numerical rating scale) was recorded and symptom of local anaesthesia was monitored. On $1^{\text {st }}$ post operative day, the NRS score was 3 on rest and 4 on flexion of hip. There was no symptom of local anaesthesia toxicity. We also added tab ultracet, combination of tramadol $37.5 \mathrm{mg}$ and
DOI: http://dx.doi.org/10.3126/ jnhrc.v16i2.20320
Correspondence: Dr Bigen man Shakya, Department of Anaesthesiology Tribhuvan university Teaching hospital, Maharajgunj, Kathmandu, Nepal. Email: bigensk@ yahoo.com. 
paracetamol 325mg. The NRS further decreased with addition of above drug. The infusion was continued for 72 hours without any complication and patient was sent to ward after 72 hours with Tab ultracet.

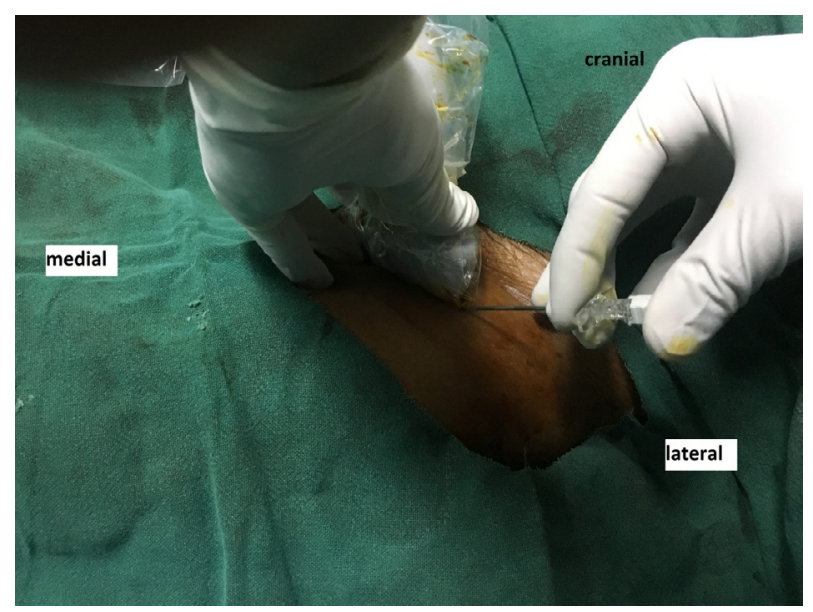

Figure1. USG probe placed transversely on upper thigh below inguinal ligament and touhy needle used for fascia iliaca block.

\section{DISCUSSION}

The postoperative management of pain of elderly patient is challenging. With increasing age, the co -morbidity increases and so does the polypharmacy. The drug interaction also has to be kept in mind. The initial step of pain management is pain assessment. In cognitively intact geriatric patients the VAS(visual analoge scale) as well as NRS(Numerical rating scale) for pain assesment is well accepted. ${ }^{1}$ The problem arises when the patient has dementia. In those cases behavioral scales have to be used. Another common misconception among the health professionals is that geriatic patients experience less pain and have higher pain tolerance in compare to young. It has been proved that this is not the case and it is because these patients complain less. ${ }^{2}$ The better way is to ask if he/she has pain or not. The another common problem is the change in both pharmacokinetics and pharmacodynamics with age. The pharmacokinetics and pharmocodynamic of many drugs are not well defined because of exclusion of old age in drug trials. ${ }^{3}$

The method available for the postoperative pain management in our institute are IV opioid, IV /oral paracetamol, IV /oral Nsaids, epidural, regional block. NSAIDS is contraindicated in this patient due to risk of nephrotoxicity. Though the opioid is still the gold standard for management of acute postoperative pain because of old age, the complication like respiratory depression is relatively high. Epidural is one of the options but we wanted to avoid undetected hypotension which may further compromise the renal function. The best option would be Regional block followed by continuous infusion with local anaesthetic agent. Paracetamol in analgesic dose is now well accepted for post operative pain management including orthopaedic surgeries. ${ }^{4}$

The nerve supply to lower extremity is provided by femoral nerve, lateral cutaneous nerve of thigh, obturator and sciatic nerve. The femoral nerve gives sensory supply to the anteromedial surface of the thigh and the medial aspect of the lower leg, ankle and foot via its terminal branch, the saphenous nerve. The lateral cutaneous nerve gives sensory supply to the lateral aspect of the thigh as far distal as the knee. The regional technique available for upper portion of lower limb are femoral nerve block, 3 in 1 block and fascial iliaca block. The fascia iliaca compartment block is a method to provide peri-operative analgesia in patients with painful conditions affecting the thigh, the hip joint and/or the femur. It requires low skill and even practiced by non anaesthesiologist and junior registars with good results. ${ }^{5}$

Fascia lliaca compartment is a potential space formed anteriorly by the posterior surface of the fascia iliaca, which covers the iliacus muscle and, with a medial reflection, every surface of the psoas major muscle and posteriorly by the anterior surface of the iliacus muscle and the psoas major muscle. This compartment block allow femoral and later cutaneous nerve to be blocked reliably. The sciatic nerve is not blocked. There was inconsistent results with respect to distribution of local anaesthetic and effect on obturator nerve. Recently, Swenson et al showed with both ultrasound as well as MRI that there was no local anaesthetic distribution to obturator nerve and hence nerve block did not occur.$^{6}$

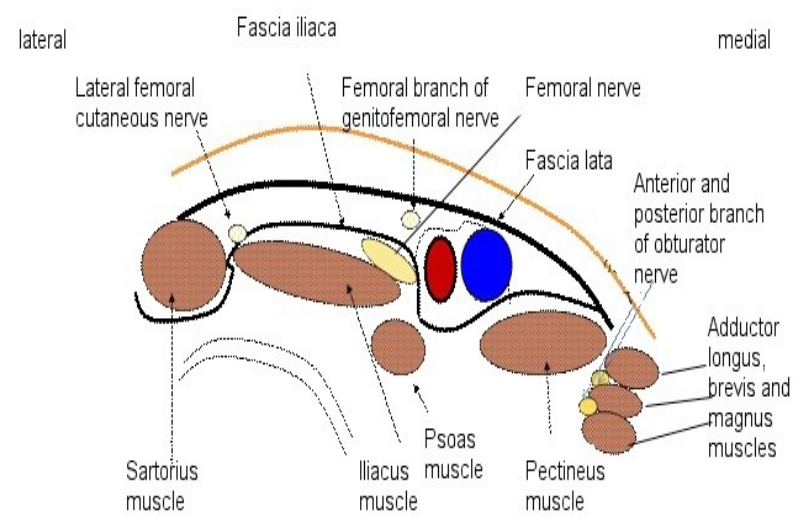

Figure 2. Cross section of right thigh just below anterior superior iliac spine.

With the use of Ultrasound, under image guidance the needle is advanced beneath fascia iliaca. 


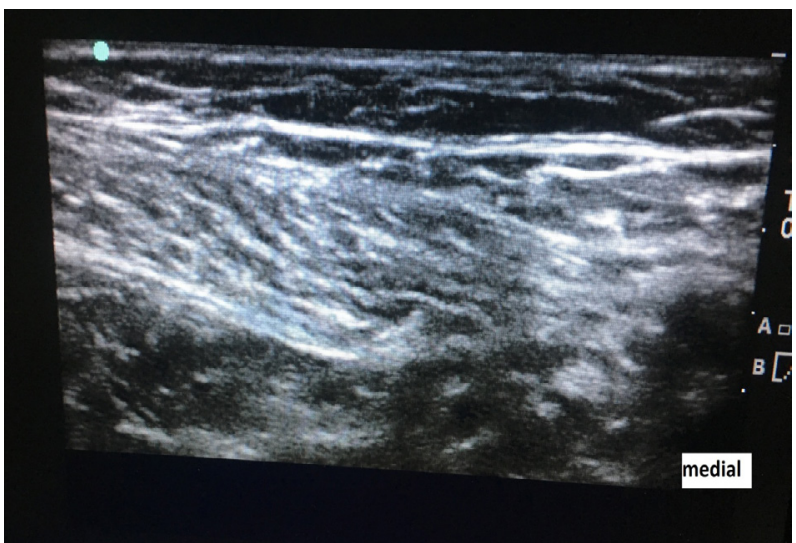

Figure 3. Sonoanatomy of upper thigh region.

The ultrasound increases the frequency of sensory loss in medial aspect of thigh. ${ }^{7}$ Ultrasound guided continous fascia iliaca compartment block is comparable with continous femoral nerve block for fracture neck of femur in regard to analgesia. ${ }^{8}$ The insertion of catheter allows continous infusion of local anaesthetic. The $0.25 \%$ Ropivacine has been used at rate of $0.1 \mathrm{ml} / \mathrm{kg} / \mathrm{hr}$ for analgesia effectively without any adverse effects. ${ }^{9}$

Fascia lliaca compartment block only allows effective analgesia not anaesthesia. The most common indication is fracture of femur and postoperative analgesia after above knee amputation.

\section{CONCLUSIONS}

The pain management can be achieved by use of continous regional block as part of multi modal regime. The continuous fascia iliaca compartment block is safe as well as effective and should be brought in to our practice also. The main aim of this case report to encourage its use in our country.

\section{REFERENCES}

1. AGS Panel on Persistent Pain in Older Persons. The management of persistent pain in older persons. J Am Geriatr Soc. 2002;50(6):S205-S224.[PubMed]

2. Hadjistavropoulos $T$, Herr $K$, Turk DC et al. An interdisciplinary J. Pain23expert consensus statement on assessment of pain in older person. Clin J Pain.2007(1):S1e43.[PubMed]

3. Van Spall HG, Toren A, Kiss A, Fowler RA. Eligibility criteria of randomized controlled trials published in highimpact general medical journals: a systematic sampling review. JAMA. 2007;297(11):1233-1240.[Full Text]
4. Sinatra R, Jahr J, Reynold L, Viscusi E, Groudine S, PayenChampenois C. Efficacy and safety of single and repeated administration of 1 gram intravenous acetaminophen injection (paracetamol) for pain management after major orthopedic surgery. Anesthesiology. 2005;102(4):822 831.[Full Text]

5. Hogh A, Dremstrup L, Jensen S S, Lindholt J. Fascia iliaca compartment block performed by junior registrars as a supplement to pre-operative analgesia for patients with hip fracture. Strat Traum Limb Recon. 2008;3:65-70 [DOI]

6. Swenson JD et al. Local anesthetic injection deep to the fascia iliaca at the level of the inguinal ligament : the pattern of distribution and effects on the obturator nerve.J Clin Anesth. 2015;27(8):652-7. doi: 10.1016/j. jclinane.2015.07.001.

7. Dolan J et al. Ultrasound guided fascia iliaca block:a comparison with the loss of resistance technique. Reg Anesth Pain Med. 2008;33(6):526-31.[PubMed]

8. Yu Bi et al. Ultrasound-guided continuous femoral nerve block vs continuous fascia iliaca compartment block for hip replacement in elderly. Medicine. 2016;95:42. [DOI]

9. H Nie, YX Yang, Y Wang, Y liu,B Zhao, B luan. Effects of continuous fascia iliaca compartment blocks for postoperative analgesia in patients with hip fracture. Pain Res Mang. 2015;20(4):210-212.[Full Text] 